\title{
Exposição à violência em adolescentes de diferentes contextos: família e instituições
}

\author{
Luiza Lima Braga \\ Débora Dalbosco Dell'Aglio \\ Universidade Federal do Rio Grande do Sul
}

\begin{abstract}
Resumo
Este estudo investigou a frequência da exposição à violência intrafamiliar e extrafamiliar, em 946 adolescentes com idades entre 12 e 19 anos $(M=15,42 ; D P=1,67)$, que viviam em diferentes contextos: com as famílias (G1), em instituições para cumprimento de medidas socioeducativas (G2) e em instituições de acolhimento (G3). Foi utilizado o Questionário da Juventude Brasileira. Foi observado que os adolescentes de G3 apresentaram maior frequência de exposição à violência intrafamiliar, enquanto os adolescentes de G2 à violência extrafamiliar, sendo as meninas as vítimas mais frequentes em todos os contextos. A importância de prevenir a exposição dos jovens à violência é destacada, bem como a necessidade de promover intervenções com os adolescentes em acolhimento institucional, já que este grupo mostrou-se o mais vulnerável.
\end{abstract}

Palavras-chave: adolescentes; violência intrafamiliar; violência extrafamiliar.

\begin{abstract}
Exposure to violence on adolescents from different contexts: family and institutions. This study verified the frequency of the exposure to domestic and community violence, on 946 adolescents with ages ranging from 12 to 19 years old $(M=15.42 ; S D=1.67)$, who were from different contexts: with their families (G1); young offenders who were deprived of freedom (G2); and adolescents living in shelters (G3). They completed the Brazilian Youth Questionnaire. Findings indicated that adolescents of G3 showed higher levels of exposure to domestic violence and adolescents of G2 were more exposed to community violence. Furthermore, the females were the most frequent victims in all contexts. The importance of preventing the youth exposure to violence is highlighted, as well as promoting interventions with adolescents who live in shelters because this group seems to be the most vulnerable.
\end{abstract}

Keywords: adolescents; domestic violence; community violence.

A violência contra crianças e adolescentes, devido às consequências psicossociais que gera, tem sido considerada um problema de saúde pública que compromete a saúde e a qualidade de vida das pessoas, tornandoas mais vulneráveis. Com relação à criança e ao adolescente, é uma grave violação de direitos, impossibilitando-os de se desenvolverem em condições saudáveis (Pesce, 2009).

A violência é classificada como o uso intencional da força física ou poder, que pode resultar em ferimentos, morte, dano psicológico, privação, além de prejudicar o desenvolvimento (World Health Organization - WHO, 2010). Muitos estudos têm verificado que indivíduos expostos a diferentes formas de violência ao longo de seu desenvolvimento apresentam risco aumentado para o surgimento de sintomas ou transtornos psicológicos, como por exemplo: baixa autoestima, baixo senso de autoeficácia, comportamento agressivo, sintomas internalizantes e externalizantes (Sullivan, Farrell, Kliewer, Vulin-Reynolds, \& Valois, 2007), podendo ocasionar quadros psicopatológicos como depressão e ansiedade (MacMillan et al., 2001), Transtorno de Estresse Pós-Traumático (Fowler, Tompsett, Braciszewski, Jacques-Tiura, \& Baltes, 2009; Kunst, Winkel, \& Bogaerts, 2010; McDonald \& Richmond, 2008), Transtorno de Déficit de Atenção e Hiperatividade (Vasconcelos et al., 2005), transtornos alimentares (Narvaz \& Oliveira, 2009), comportamento suicida (Espinoza-Gomez et al., 2010), entre outros.

Dentre as muitas formas de violência, a violência intrafamiliar (ou maus tratos) e a violência vivenciada em contextos extrafamiliares, como a comunidade e a escola, têm chamado a atenção da mídia, autoridades, professores e pesquisadores devido à alta prevalência e repercussões destes problemas não apenas no Brasil (Benett et al., 2006; De Antoni, 2005; Ristum, 2010), mas em diversos países (Sullivan et al., 2007).

A Organização Mundial da Saúde (WHO, 2010) define os maus tratos como todo tipo de abuso físico ou emocional, 
negligência ou exploração comercial, que resulta em um dano atual ou potencial para a saúde, desenvolvimento, dignidade e sobrevivência da criança ou adolescente e ocorre no contexto de um relacionamento de responsabilidade, confiança e poder. O tipo mais frequente de maus-tratos contra a criança ou adolescente é a violência doméstica, que ocorre na maioria das vezes dentro dos lares ou no convívio familiar. No Brasil, a alta prevalência da violência intrafamiliar se constitui em sério problema de saúde e em obstáculo para o desenvolvimento social e econômico, além de denunciar a violação dos direitos humanos (Brasil, 2002).

Embora esforços tenham sido feitos no sentido de obter dados sobre a frequência da ocorrência de maus tratos contra crianças e adolescentes, não há uma estimativa global confiável sobre a prevalência destas situações (WHO, 2010). Os serviços de saúde encontram dificuldades para diagnosticar e registrar casos de violência nas famílias (Brasil, 2002). Entretanto, dados da Organização Mundial da Saúde (WHO, 2010) estimam que aproximadamente $20 \%$ das mulheres, e cerca de 5 a $10 \%$ dos homens, relatam ter sido sexualmente abusados quando crianças, enquanto 25 a 50\% das crianças relatam história de abuso físico. Já a violência psicológica (ou abuso emocional) no âmbito familiar ainda é pouco estudada, apesar de trazer inúmeras consequências negativas para as crianças e adolescentes vítimas (Brodski, 2010). No estudo de Brodski (2010), com universitários, foi encontrada correlação negativa entre abuso emocional e autoestima, afeto positivo e satisfação de vida. Este tipo de violência não tem limites culturais, sociais, ideológicos ou geográficos e está envolta por um pacto de silêncio, que torna difícil o diagnóstico (Abranches \& Assis, 2011).

Também tem sido verificado que muitos jovens estão expostos a diferentes tipos de violência fora do âmbito familiar, principalmente na comunidade, sendo de alta frequência a exposição de adolescentes à violência extrafamiliar (Benetti et al., 2006). A Organização Mundial da Saúde (WHO, 2010) define a violência comunitária como episódios envolvendo violência ocorridos no contexto social/ambiental de inserção do indivíduo, incluindo agressões, estupros, assaltos, roubos, homicídios, presença de armas e tráfico de drogas. Este tipo de violência engloba a vitimização direta e também o testemunho da violência contra outros (McDonald \& Richmond, 2008).

Alguns estudos norte-americanos têm investigado o impacto de uma comunidade violenta em adolescentes. Sullivan et al. (2007) verificaram que a exposição à violência na comunidade está relacionada a numerosos resultados negativos para os jovens, incluindo o aumento da frequência de comportamentos externalizantes, tais como o uso de drogas e agressão. McDonald e Richmond (2008) observaram uma forte relação entre exposição à violência comunitária e sintomas de estresse pós-traumático e agressão. Além disso, foram identificados sintomas de ansiedade, depressão e ideação suicida nos adolescentes expostos à violência. No estudo de Fowler et al. (2009) foi verificada uma associação consistente entre violência comunitária, sintomas de estresse pós-traumático e problemas externalizantes.

No contexto brasileiro, encontram-se poucos estudos específicos sobre exposição à violência extrafamiliar no período da adolescência (Benetti et al., 2006; De Antoni et al., 2011; Polanczyk et al., 2003; Sá, Curto, Bordin, \& Paula, 2009), e a maioria deles vem sendo realizado em contextos escolares (Guzzo, 2001; Lisboa \& Koller, 2004; Lisboa, 2005; Ristum, 2010). Considerando que diferentes contextos podem oferecer condições variadas para o desenvolvimento, o objetivo deste estudo foi observar a exposição à violência intra e extrafamiliar em adolescentes que vivem em diferentes contextos.

\section{Método}

\section{Participantes}

Participaram deste estudo 946 adolescentes com idades entre 12 e 19 anos $(M=15,42 ; D P=1,67)$, sendo $53,4 \%$ do sexo feminino e $46,6 \%$ do sexo masculino, que estavam inseridos nos seguintes contextos: Grupo 1, 691 adolescentes que estudavam entre a $6^{\underline{a}}$ série do Ensino Fundamental e o $3^{\underline{0}}$ ano do Ensino Médio, em escolas públicas da cidade de Porto Alegre/RS e que viviam com suas famílias; Grupo 2, 142 adolescentes em conflito com a lei que cumpriam medidas socioeducativas de internação ou internação provisória na Fundação de Atendimento Socioeducativo (FASE); e Grupo 3, 113 adolescentes que estavam sob proteção em instituições governamentais de acolhimento, municipais (Fundação de Assistência Social e Cidadania- FASC), estaduais (Fundação de Proteção Especial) e não governamentais (ONGs conveniadas com a prefeitura de Porto Alegre). Tais grupos foram compostos de forma a se investigar o fenômeno da violência em contextos diferenciados de desenvolvimento de adolescentes. A Tabela 1 apresenta os dados sociodemográficos dos participantes por grupo.

\section{Instrumento}

Os participantes responderam ao Questionário da Juventude Brasileira (Dell'Aglio, Koller, Cerqueira-Santos, \& Colaço,

Tabela 1

\begin{tabular}{|c|c|c|c|}
\hline & G1-Familia & G2 - Fase & G3-Abrigos \\
\hline Sexo & $\begin{array}{l}\text { Meninos - 38,9\% } \\
\text { Meninas - } 61,1 \%\end{array}$ & $\begin{array}{l}\text { Meninos - } 89,4 \% \\
\text { Meninas - } 10,6 \%\end{array}$ & $\begin{array}{l}\text { Meninos - 39,8\% } \\
\text { Meninas - 60,2\% }\end{array}$ \\
\hline Idade & $\begin{array}{l}M=15,16 \\
D P=1,56\end{array}$ & $\begin{array}{l}M=15,16 \\
D P=1,56\end{array}$ & $\begin{array}{l}M=14,85 \\
D P=1,43\end{array}$ \\
\hline Série & $\begin{array}{c}6^{\mathrm{a}}, 7^{\mathrm{a}} \text { e } 8^{\mathrm{a}} \text { série }-47,2 \% \\
1^{\circ} \text { e } 2^{\mathrm{o}} \text { ano }-50,3 \% \\
3^{\mathrm{o}} \text { ano }-2,5 \%\end{array}$ & $\begin{array}{c}1^{\mathrm{a}} \text { a } 4^{\mathrm{a}} \text { série }-5,9 \% \\
5^{-\mathrm{a}}, 6^{\mathrm{a}} \text { e } 7^{\mathrm{a}} \text { série }-63,7 \% \\
8^{\mathrm{a}} \text { série e } 1^{\circ} \text { ano }-21,5 \% \\
2^{-} \text {e } 3^{-} \text {ano }-8,9 \%\end{array}$ & $\begin{array}{c}1^{\mathrm{a}^{\mathrm{a}}} \text { a } 4^{-\mathrm{a}} \text { série }-30 \% \\
5^{\mathrm{a}}, 6^{\mathrm{a}} \text { e } 7^{-\mathrm{a}} \text { série }-54,4 \% \\
8^{\mathrm{a}} \text { série e } 1^{\mathrm{o}} \text { ano }-10,2 \% \\
2^{-} \text {ano }-0,9 \% \\
\text { EJA }-4,5 \%\end{array}$ \\
\hline
\end{tabular}


2011), composto por 77 questões que investigam fatores de risco e proteção no desenvolvimento, derivadas de escalas já usadas em estudos anteriores (ver Libório \& Koller, 2009). Para este estudo foram consideradas apenas as variáveis sociodemográficas e as questões 31 e 62 que investigavam especificamente a exposição a diferentes tipos de violência familiar e extrafamiliar, dentre os seguintes: a) ameaça ou humilhação; b) soco ou surra; c) agressão com objetos; d) alguém ter mexido no corpo do jovem sem a vontade do mesmo; e) relação sexual forçada.

\section{Procedimentos}

Os aspectos éticos que garantem a integridade dos participantes foram assegurados, tendo como base a Resolução $\mathrm{n}^{\mathrm{o}} 196$ (Conselho Nacional de Saúde, 1996). O projeto de pesquisa foi aprovado pelo Comitê de Ética em Pesquisa da Universidade, sob protocolo número 2009060. Inicialmente, foi realizado o contato com as instituições responsáveis pelos adolescentes solicitando concordância para a realização do estudo. Foi também solicitado o Termo de Consentimento Livre e Esclarecido aos pais ou às instituições (nos casos em que estas mantinham a guarda dos adolescentes).

Especificamente para a composição do Grupo 1 foi constituída uma amostra aleatória por conglomerados através de sorteio das escolas públicas municipais e estaduais da cidade de Porto Alegre e, posteriormente, sorteio das turmas de adolescentes em cada escola selecionada, de forma que a amostra pudesse ser representativa dos adolescentes estudantes de escolas públicas. O número de participantes foi obtido através do cálculo amostral, a partir do número total de alunos de ensino fundamental e ensino médio matriculados em escolas públicas de Porto Alegre, com uma margem de erro estabelecida de 4\% (Barbetta, 2001). Foram coletados dados em 12 escolas, com uma média de 50 adolescentes em cada uma. Para acessar os adolescentes do Grupo 2, inicialmente foi realizado contato com a administração central da FASE e posteriormente com os monitores de cada unidade. Os adolescentes responderam a pesquisa em grupos de 6 a 8 jovens, provenientes de cinco unidades: Internação Provisória, POA I, POA II, Padre Cacique (adolescentes vindos do interior) e CASEF (unidade feminina). Foram convidados a participar do estudo todos os adolescentes destas unidades que tivessem cursado pelo menos a quinta série do Ensino Fundamental. Segundo a Assessoria de Informação e Gestão da FASE-RS, no período de coleta de dados, havia 565 meninos internados na instituição e 31 meninas, demonstrando que a amostra representou, respectivamente, $23 \%$ dos meninos e $48 \%$ das meninas em cumprimento de medida em regime fechado.

Para a composição do Grupo 3, foram considerados os seguintes critérios de inclusão na amostra: adolescentes que tinham condições cognitivas para responder ao instrumento da pesquisa, de acordo com a avaliação dos cuidadores, e que estivessem há pelo menos 30 dias em acolhimento institucional. Todos os adolescentes que estavam em acolhimento nas instituições contatadas durante o período de coleta de dados foram convidados a participar do estudo. Dessa forma, a amostragem foi não probabilística, mas tendo em vista que representou $59 \%$ do total de adolescentes que possuíam os critérios estipulados na rede de acolhimento governamental e não governamental conveniada, no período de coleta, estima-se que seja representativa dos jovens em acolhimento.

Os adolescentes dos três grupos foram convidados a participar, esclarecendo a voluntariedade da participação, a garantia de sigilo das informações pessoais e a possibilidade de desistência a qualquer momento. Os adolescentes que concordaram em participar também assinaram o Termo de Consentimento Livre e Esclarecido. O instrumento foi aplicado nas próprias escolas, abrigos e nas unidades da FASE, com duração de aproximadamente 60 minutos. Foi disponibilizada a assistência por parte da equipe de pesquisa, que foi capacitada para este tipo de coleta de dados, no caso de participantes que necessitaram de apoio durante ou imediatamente após a realização da coleta de dados ou no caso de serem identificadas situações de risco iminente.

\section{Análise de dados}

Os dados do instrumento foram digitados em uma tabela do programa SPSS, a partir da qual foram realizadas análises descritivas, observando-se frequências, médias e desvios-padrão das variáveis investigadas. Também foram realizados testes de Qui-Quadrado para verificar as associações dos tipos de violência com os grupos e diferenças por sexo.

\section{Resultados}

\section{Violência intrafamiliar}

Os resultados mostraram diferenças entre os três grupos investigados quanto à exposição aos diferentes tipos de violência intrafamiliar, conforme pode ser observado na Tabela 2. O grupo dos adolescentes que viviam em instituições de proteção (G3) mostrou-se, de maneira geral, o grupo que mais havia experienciado situações de violência no ambiente familiar, seguido do grupo de adolescentes que cumpriam medidas socioeducativas (G2) e dos adolescentes que viviam com a família (G1). Foram observadas diferenças significativas entre o grupo dos adolescentes sob proteção (G3) e os outros dois grupos, em todos os tipos de violência. Além disso, destaca-se também a incidência da violência física, através de soco ou surra, tanto no G2 como no G3.

Com relação à frequência de violência intrafamiliar por sexo e por grupo, foi possível constatar que as meninas pertencentes ao grupo dos adolescentes em situações de acolhimento institucional (G3) apresentaram as frequências mais altas de exposição à violência, tanto quando comparadas aos adolescentes masculinos do mesmo grupo quanto quando comparadas às meninas que viviam com suas famílias (G1) e que estavam em cumprimento de medidas socioeducativas (G2). Foram observadas diferenças estatisticamente significativas entre o grupo das meninas em situação de acolhimento institucional e os outros grupos. Também foi observada diferença por sexo em cada grupo, tendo sido encontrada diferença significativa apenas no G3 em ameaça ou humilhação $\left(\chi^{2}=7,91 ; g l=1 ; p=0,005\right)$, com frequências mais altas entre as meninas. Foi verificado que os meninos de G1 relataram frequências mais altas de exposição a todos os tipos de 
Tabela 2

Percentuais de exposição à violência intrafamiliar, por grupo

\begin{tabular}{lccccc}
\hline \multicolumn{1}{c}{ Tipo de violência } & $\begin{array}{c}\mathrm{G} 1 \\
\text { (Família) }\end{array}$ & $\begin{array}{c}\mathrm{G} 2 \\
\text { (Fase) }\end{array}$ & $\begin{array}{c}\text { G3 } \\
\text { (Abrigos) }\end{array}$ & $\chi^{2}$ & \multicolumn{1}{c}{$p$} \\
\hline Ameaça ou humilhação & 20,1 & 15,5 & $33(+)$ & 12,72 & 0,002 \\
Soco ou surra & $24,5(-)$ & $50,4(+)$ & $50,5(+)$ & 56,21 & $<0,001$ \\
Agressão com objetos & $20,8(-)$ & $35,3(+)$ & $52(+)$ & 52,44 & $<0,001$ \\
Mexer no corpo & $2,1(-)$ & 4,4 & $21,7(+)$ & 79,48 & $<0,001$ \\
Relação sexual forçada & $0,7(-)$ & 1,5 & $16,5(+)$ & 88,27 & $<0,001$ \\
\hline
\end{tabular}

$(+)$ resíduos ajustados $>1,96 ;(-)$ resíduos ajustados $<-1,96$

violência do que as meninas do mesmo grupo, com exceção de "mexer no corpo contra a vontade". Em contrapartida, observouse que as meninas do G2 apresentaram frequências maiores de exposição a todos os tipos de violência do que os meninos deste mesmo grupo, com exceção de "agressão com objetos", conforme pode ser observado na Tabela 3 .

\section{Violência extrafamiliar}

Este estudo também investigou a exposição à violência em contextos não familiares. Os resultados mostraram diferenças entre os grupos investigados quanto à frequência da exposição à violência extrafamiliar. Foi possível verificar que os adolescentes de G2 apresentaram frequências mais altas de exposição à

Tabela 3

\begin{tabular}{|c|c|c|c|c|c|c|c|}
\hline Tipo & Sexo & $f$ & $\begin{array}{c}\mathrm{G} 1 \\
\text { (Família) }\end{array}$ & $\begin{array}{c}\mathrm{G} 2 \\
\text { (Fase) }\end{array}$ & $\begin{array}{c}\mathrm{G} 3 \\
\text { (Abrigos) }\end{array}$ & $\chi^{2}$ & $p$ \\
\hline \multirow{2}{*}{$\begin{array}{l}\text { Ameaça ou } \\
\text { humilhação }\end{array}$} & $\mathrm{M}$ & 84 & 21,2 & 15 & 17,8 & 2,22 & 0,32 \\
\hline & $\mathrm{F}$ & 114 & $19,4(-)$ & 20 & $43,4(+)$ & 18,85 & $<0,01$ \\
\hline \multirow{2}{*}{ Soco ou surra } & M & 156 & $29,8(-)$ & $50(+)$ & 35,7 & 14,91 & 0,001 \\
\hline & $\mathrm{F}$ & 135 & $21,2(-)$ & $53,3(+)$ & $60(+)$ & 48,14 & $<0,01$ \\
\hline \multirow{2}{*}{$\begin{array}{l}\text { Agressão com } \\
\text { objetos }\end{array}$} & M & 125 & $24,2(-)$ & $37,2(+)$ & 37,2 & 8,20 & 0,017 \\
\hline & $\mathrm{F}$ & 120 & $18,6(-)$ & 20 & $61,5(+)$ & 56,68 & $<0,01$ \\
\hline \multirow{2}{*}{$\begin{array}{l}\text { Mexer no corpo } \\
\text { contra a vontade }\end{array}$} & M & 8 & 1,1 & 1,7 & $7,1(+)$ & 7,16 & 0,028 \\
\hline & F & 35 & $2,7(-)$ & $26,7(+)$ & $31,3(+)$ & 77,90 & $<0,01$ \\
\hline \multirow{2}{*}{$\begin{array}{l}\text { Relação sexual } \\
\text { forçada }\end{array}$} & M & 9 & 0,8 & 0 & $17,1(-)$ & 49,46 & $<0,01$ \\
\hline & F & 15 & $0,7(-)$ & $13,3(+)$ & $16,1(+)$ & 48,85 & $<0,01$ \\
\hline
\end{tabular}

$(+)$ resíduos ajustados $>1,96 ;(-)$ resíduos ajustados $<-1,96$

violência na maioria dos tipos de violência, seguido de G3 e G1. Entretanto, com relação aos tipos de violência "mexer no corpo contra a vontade" e "relação sexual forçada" os adolescentes de G2 apresentaram frequências menores de exposição do que os outros dois grupos, como mostra a Tabela 4.

Com relação à frequência de violência extrafamiliar por sexo e por grupo, foi possível constatar que no G1, os meninos apresentaram frequências mais altas do que as meninas em três

Tabela 4

Percentuais de exposição à violência extrafamiliar, por grupo

\begin{tabular}{lccccc}
\hline \multicolumn{1}{c}{ Tipo de violência } & $\begin{array}{c}\text { G1 } \\
\text { (Família) }\end{array}$ & $\begin{array}{c}\text { G2 } \\
\text { (Fase) }\end{array}$ & $\begin{array}{c}\text { G3 } \\
\text { (Abrigos) }\end{array}$ & $\chi^{2}$ & $p$ \\
\hline Ameaça ou humilhação & 40 & 50 & 45,5 & 5,13 & 0,07 \\
Soco ou surra & 20,1 & 52,8 & 35,5 & 63,60 & 0,001 \\
Agressão com objetos & 7 & 36 & 20 & 87,52 & 0,001 \\
Mexer no corpo & 5 & 4,1 & 10 & 5,31 & 0,07 \\
Relação sexual forçada & 0,9 & 0 & 8,3 & 33,57 & 0,001 \\
\hline
\end{tabular}

dos cinco tipos de violência investigados, visto que nas duas questões sobre abuso sexual "mexer no corpo contra a vontade" e "relação sexual forçada", as meninas tiveram maior exposição. Entretanto, no G2 e G3 as meninas estiveram mais expostas à violência do que os meninos. Especificamente no G2 as meninas tiveram maior exposição à violência do que os meninos, com exceção de "agressão com objetos". Não foi possível comparar as meninas e meninos deste grupo na questão "relação sexual forçada", pois nenhum caso foi verificado. Com relação ao G3, as meninas apresentaram frequências mais altas do que os meninos em todos os tipos de violência, com exceção de "soco ou surra", conforme mostra a Tabela 5 .

\section{Discussão}

Esta pesquisa investigou a exposição à violência intrafamiliar e extrafamiliar em adolescentes que vivem em diferentes contextos: com suas famílias (G1), em instituições para cumprimento de medidas socioeducativas (G2) e em instituições de acolhimento (G3). Os resultados indicaram que os adolescentes do G3 estiveram mais expostos à violência intrafamiliar, enquanto os adolescentes do G2 à violência 
Tabela 5

Percentuais de ocorrência de violência extrafamiliar por sexo e por grupo

\begin{tabular}{lccccccc}
\hline \multicolumn{1}{c}{ Tipo } & Sexo & $f$ & $\begin{array}{c}\text { G1 } \\
\text { (Família) }\end{array}$ & $\begin{array}{c}\text { G2 } \\
\text { (FASE) }\end{array}$ & $\begin{array}{c}\text { G3 } \\
\text { (Abrigos) }\end{array}$ & $\chi^{2}$ & $p$ \\
\hline Ameaça/Humilhação & M & 202 & 49 & 48,3 & 39,5 & 1,35 & 0,05 \\
Soco ou Surra & $\mathrm{F}$ & 182 & $33,7(-)$ & 61,5 & $49,3(+)$ & 9,59 & 0,008 \\
& $\mathrm{M}$ & 163 & $33,3(-)$ & $52,6(+)$ & 39,5 & 12,34 & 0,002 \\
Agressão com & $\mathrm{F}$ & 79 & $12(-)$ & $53,8(+)$ & $32,8(+)$ & 33,28 & $<0,001$ \\
Objetos & $\mathrm{M}$ & 80 & $11,2(-)$ & $38,4(+)$ & 18,6 & 37,11 & $<0,001$ \\
Mexer no corpo & $\mathrm{F}$ & 34 & $4,3(-)$ & 15,4 & $20,9(+)$ & 26,33 & $<0,001$ \\
& $\mathrm{M}$ & 15 & 3,5 & 3,6 & 4,7 & 0,14 & 0,9 \\
Relação Sexual & $\mathrm{F}$ & 34 & $5,8(-)$ & 8,3 & $13,4(+)$ & 5,3 & 0,07 \\
Forçada & $\mathrm{M}$ & 4 & 0,8 & 0 & 2 & - & - \\
\hline
\end{tabular}

$(+)$ resíduos ajustados $>1,96 ;(-)$ resíduos ajustados $<-1,96$

extrafamiliar. Em ambos os tipos de violência investigados, os adolescentes que vivem com suas famílias (G1) apresentaram menor frequência de exposição à violência.

Os resultados deste estudo revelaram elevados e preocupantes índices de exposição à violência intrafamiliar entre os adolescentes que estão em instituições de acolhimento (G3). Embora tais adolescentes não estejam mais vivendo com suas famílias, foi possível verificar que muitos deles já haviam passado por diferentes formas de violência no âmbito familiar ao longo de seu desenvolvimento. De acordo com um levantamento nacional (Silva, 2004), os principais motivos para o acolhimento de crianças e adolescentes são: pobreza, abandono, violência doméstica, uso de drogas por parte dos pais ou responsáveis, vivência de rua, orfandade, prisão dos pais ou responsáveis e, por fim, o abuso sexual. Neste sentido, a violência a que estes jovens estiveram expostos pode ter sido um dos motivos que causaram o afastamento de suas famílias e encaminhamento para instituições de proteção, pois a literatura aponta que fatores de risco presentes na família, como a violência, podem contribuir para o ingresso de crianças e adolescentes em instituições de acolhimento (Silva, 2004; Siqueira, Betts, \& Dell'Aglio, 2006). Por outro lado, observar que estes adolescentes que foram vítimas de violência intrafamiliar se encontram em medida de proteção, atesta que os princípios propostos pelo Estatuto da Criança e do Adolescente (Brasil, 1990) estão sendo cumpridos, na medida em que está sendo oferecido um espaço alternativo, embora temporário, para seu desenvolvimento.

A literatura psicológica aponta que os meninos estão mais expostos à violência, especialmente no contexto extrafamiliar, constituindo-se num grupo de risco para testemunhar, sofrer e perpetrar atos violentos (Benetti et al., 2006) já que, por apresentarem com mais frequência comportamentos agressivos e externalizantes, envolvem-se mais em situações de lutas e brigas, roubos, crimes violentos e vandalismo (Hein, 2004). Em contrapartida, as meninas são descritas pela literatura como as maiores vítimas de violência no ambiente familiar (Brasil, 2002), principalmente no que se refere ao abuso sexual e negligência (Sullca \& Schirmer, 2006). Porém, no presente estudo, as meninas constituíram-se no grupo de maior risco tanto no contexto intra como no extrafamiliar, apresentando maior prevalência de exposição à violência, principalmente no que se refere à violência sexual. Este resultado também foi encontrado em outros estudos que mostram que as meninas são as maiores vítimas de violência sexual, tanto no contexto intra
(Santos, 2007) como no contexto extrafamiliar (De Antoni et al., 2011), e chegam a ser cerca de três vezes mais expostas a este tipo de violência do que os meninos (Araújo, 2002; Polanczyk et al., 2003; Santos, 2007). A maior vitimização das adolescentes do sexo feminino pode ser explicada pela construção social de gênero que ainda atribui às mulheres papel de desvalorização, passividade, resignação e submissão (Sullca \& Schirmer, 2006), embora mudanças tenham sido observadas neste cenário.

Os resultados também revelaram que o grupo que apresentou menor frequência de exposição à violência intra e extrafamiliar foi o grupo dos adolescentes que vivem com suas famílias (G1). Este resultado apoia outros estudos que indicam evidências consistentes de que a família, ainda nos dias de hoje, desempenha um importante papel de proteção no desenvolvimento de seus membros, principalmente no período da adolescência (Minuchim \& Fishman, 1990; Pratta \& Santos, 2007; Steinberg, 2000). Desta forma, torna-se importante a valorização da família, enquanto principal ambiente de desenvolvimento, e a necessidade de que as políticas que incentivam a convivência familiar e comunitária (Brasil, 1990) sejam de fato aplicadas, especialmente em situações em que os jovens são afastados de seus familiares. Por outro lado, destaca-se também a necessidade de trabalhos de prevenção junto às famílias, de forma que possam ser evitados problemas que levam a medidas mais drásticas, como o afastamento dos filhos, levando ao rompimento de vínculos e à institucionalização.

Foram observados altos níveis de abuso físico tanto no contexto intrafamiliar como no extrafamiliar, através de "soco ou surra" e "agressão com objetos" nos adolescentes de ambos os sexos, principalmente nos grupos G2 e G3. Por outro lado, as meninas estiveram muito mais expostas às questões de violência sexual em ambos os contextos (intra e extrafamiliar) do que os meninos, conforme já é amplamente divulgado na literatura (De Antoni et al., 2011; Polanczyk et al., 2003; Santos, 2007).

Os adolescentes de G2 apresentaram frequências elevadas de exposição ao tipo de violência intrafamiliar denominado "soco ou surra". A alta exposição à violência física em adolescentes com comportamento infrator ou antissocial já foi verificada em outros estudos (Dell'Aglio, Benetti, D'Incao, \& Leon, 2005; Meneghel, Giugliani, \& Falceto, 1998; Pesce, 2009; Sá et al., 2009; Salzinger, Feldman, Stockhammer, \& Hood, 2002), sugerindo uma associação entre punição física corporal e comportamentos antissociais na infância e adolescência. Cecconello, De Antoni e Koller (2003) argumentam que a utilização da força física na 
educação de crianças e adolescentes ainda é aceita em nossa sociedade e justificada pelos familiares como uma prática educativa e disciplinar. Neste sentido, trabalhos de prevenção e educação direcionados a pais, centrados no desenvolvimento de práticas educativas mais adequadas, fazem-se necessários.

Com relação ao contexto extrafamiliar, o grupo que apresentou frequências maiores de exposição à violência, de maneira geral, foi o grupo de adolescentes em cumprimento de medidas socioeducativas. O estudo de Benetti et al. (2006) encontrou resultado similar, mostrando que os adolescentes expostos às drogas ilícitas e com maior envolvimento com a polícia (como é o caso dos adolescentes de G2) tiveram maior exposição à violência comunitária. Pode-se levantar a hipótese de que a maior exposição à violência extrafamiliar em adolescentes de G2 se deva, além de aspectos contextuais e sociais a que estão expostos, também a algumas características de temperamento e personalidade presentes em indivíduos com comportamento antissocial ou infrator, que, segundo Hein (2004), podem apresentar comportamentos como agressividade, desobediência, rebeldia, oposicionismo, temperamento exaltado e baixo controle de impulsos. Estas características podem estar relacionadas a um maior envolvimento destes adolescentes em comportamentos de risco, atos infracionais e situações de violência no ambiente extrafamiliar, não apenas como autores de violência, mas também como vítimas, justamente por colocaram-se em situações de vulnerabilidade envolvendo diferentes tipos de violência.

Análises intragrupos permitiram observar que as adolescentes do sexo feminino de G2 estiveram mais expostas a todos os tipos de violência intrafamiliar como também de violência extrafamiliar, quando comparadas aos meninos do mesmo grupo, com exceção de "agressão com objetos", que apresentou frequências maiores entre os meninos tanto no contexto intra como no extrafamiliar. Por outro lado, nenhum adolescente de $\mathrm{G} 2$ relatou ter sofrido relação sexual forçada no ambiente extrafamiliar. Um estudo realizado com 50 meninas infratoras encontrou resultado similar, revelando a presença de violência intra e extrafamiliar no contexto em que as jovens viviam, incluindo agressões físicas, discussões verbais, ameaças e abuso sexual, envolvimento com o tráfico e uso de drogas, bem como escolhas amorosas por companheiros violentos e também envolvidos no mundo infracional (Dell'Aglio, Santos, \& Borges, 2004). Novamente a questão de gênero se faz presente em contextos de vulnerabilidade, sendo necessário que projetos de intervenção direcionados a adolescentes levem em consideração estes aspectos, buscando minimizar a exposição das meninas a este tipo de violência.

A alta exposição dos jovens à violência extrafamiliar é um dado alarmante deste estudo. Spano, Rivera e Bolland (2010) investigaram se as práticas parentais poderiam ser utilizadas para diminuir os níveis de exposição à violência entre jovens americanos, mostrando que os pais desempenham um importante papel na redução da exposição à violência, através do monitoramento do comportamento dos filhos e de práticas que desencorajam os adolescentes a se exporem em situações de risco. Em contrapartida, pais que utilizam a violência como uma forma de corrigir os comportamentos indesejáveis de seus filhos podem facilitar a exposição dos jovens à violência extrafamiliar. Nesta direção, o estudo de Abranches e Assis (2011) verificou que crianças e adolescentes vítimas de violência intrafamiliar por parte de pessoas significativas (como por exemplo, os pais ou cuidadores) tornam-se mais vulneráveis a sofrer violência em ambientes extrafamiliares, como escola, comunidade, e também nas relações de namoro. De Antoni et al. (2011) também destacam que o abuso sexual extrafamiliar contra adolescentes parece ser um precursor para a exploração sexual comercial infanto-juvenil.

\section{Conclusões}

Este estudo buscou investigar a ocorrência da exposição à violência intrafamiliar e extrafamiliar em adolescentes de três diferentes contextos. A partir da pesquisa realizada, foi possível verificar a ocorrência de violência na trajetória de vida de muitos dos adolescentes que viviam com suas famílias, que cumpriam medidas socioeducativas e que estavam em instituições de acolhimento, indicando que a exposição a diferentes tipos de violência é frequente na maioria dos contextos de desenvolvimento dos adolescentes. Neste sentido, a partir dos resultados apresentados, foi verificado que a exposição dos jovens à violência, tanto no ambiente intrafamiliar quanto no ambiente extrafamiliar, é de alta frequência. Este dado sugere que, apesar da existência de uma legislação no Brasil, no sentido de proteção integral à criança e ao adolescente, através do Estatuto da Criança e do Adolescente (Brasil, 1990), pode ser observada violência intrafamiliar e extrafamiliar no desenvolvimento de adolescentes, o que demonstra que os pressupostos legais não garantem a proteção dessa população. Desse modo, pode-se pensar que a violência na adolescência não é uma questão que diz respeito apenas à família das pessoas envolvidas, mas constitui-se em grave problema de saúde pública, que deve ser observado em todos os contextos de inserção dos jovens.

O presente estudo apresenta algumas limitações. Com relação ao G1, entende-se que pelo tamanho da amostra e pela característica representativa e aleatória da população adolescente matriculada em escolas públicas, os dados encontrados podem ser generalizados para esta população. Entretanto, os dados desta pesquisa não abrangem a população de adolescentes estudantes de escolas privadas. Embora não invalide os resultados, tal limitação deve ser considerada, pois os resultados poderiam ter sido mais diversificados se o estudo tivesse sido ampliado para os adolescentes de escolas particulares. Quanto aos outros grupos, embora as amostras não tenham sido aleatórias, o percentual de adolescentes acessados através da pesquisa foi representativo.

Outras dificuldades desta pesquisa podem ser apontadas. Dentre elas, destaca-se a questão ética com relação ao Termo de Consentimento Livre e Esclarecido (TCLE) solicitado aos pais dos adolescentes de G1. A exigência do TCLE pode ocasionar um viés nos resultados, pois famílias com dinâmicas de violência, ao tomarem conhecimento dos objetivos da pesquisa, podem ter encontrado receio em permitir a participação de seu filho(a) na pesquisa, negando a participação do mesmo. Sendo assim, a maioria dos participantes de G1 teriam sido, provavelmente, oriundos de famílias sem dinâmicas de violência. Entretanto, 
estas dificuldades éticas com relação ao TCLE são frequentes em estudos com crianças e adolescentes vítimas de violência (Lisboa, Habigzang, \& Koller, 2007) e não invalidam os dados deste estudo.

As diferenças evidenciadas quanto ao tipo de violência experienciada pelos adolescentes em cada contexto revelaram a importância da realização de investigações que considerem as características de adolescentes sempre de forma contextualizada. Um contexto de desenvolvimento adequado pode contribuir na redução do envolvimento dos adolescentes em situações de conflito e violência, e, sendo assim, a família, as instituições de acolhimento e as instituições para cumprimento de medidas socioeducativas devem ser ambientes de proteção, servindo como mediadores para um desenvolvimento mais adaptativo. Neste sentido, torna-se importante também a proposição de estudos longitudinais, que possam avaliar as consequências da exposição à violência no desenvolvimento dos jovens, assim como trabalhos de intervenção junto às famílias, que possam fomentar o estabelecimento de relações familiares mais positivas e saudáveis.

Por fim, compreende-se que as experiências vividas com a família e com a comunidade constituem-se em padrões de referência para os adolescentes, auxiliando-os a estabelecer suas crenças e valores sobre o mundo e sobre si mesmos, o que poderá ser positivo ou não para um desenvolvimento saudável, dependo das características desses ambientes. Desta forma, torna-se urgente a necessidade de capacitação de profissionais da área da saúde e educação para atuar em situações de violência, tanto com as crianças e adolescentes vítimas como também com suas famílias, a fim de interromper uma possível multigeracionalidade dos atos violentos e diminuir a vulnerabilidade nesta população.

\section{Referências}

Abranches, C. D., \& Assis, S. G. (2011). A (in)visibilidade da violência psicológica na infância e adolescência no contexto familiar. Cadernos de Saúde Pública, 27(5), 843-854.

Araújo, M. F. (2002). Violência e abuso sexual na família. Psicologia em Estudo, 7, 3-11.

Barbetta, P. A. (2001). Estatística aplicada às ciências sociais. Florianópolis: UFSC.

Benetti, S. P. C., Gama, C., Vitolo, M., Silva, M. B., D’Avila, A., \& Zavaschi, M. L. (2006). Violência comunitária, exposição às drogas ilícitas e envolvimento com a lei na adolescência. Psico, 37(3), 279-286.

Brasil (1990). Estatuto da criança e do adolescente. Diário Oficial da União. Lei nº 8.069, de 13 de Julho de 1990. Brasília, DF.

Brasil (2002). Violência intrafamiliar: orientações para a prática em serviço. Cadernos da Atenção Básica, $\mathrm{n}^{\circ}$ 8. Ministério da Saúde, Brasília, DF.

Brodski, S. K. (2010). Abuso emocional: suas relações com autoestima, bem-estar subjetivo e estilos parentais em universitários. (Dissertação de mestrado). Universidade Federal do Rio Grande do Sul, RS. (000760983)

Cecconello, A. M., De Antoni, C., \& Koller, S. H. (2003). Práticas educativas, estilos parentais e abuso físico no contexto familiar. Psicologia em Estudo, $8(2), 45-54$.

Conselho Nacional de Saúde (1996). Diretrizes e normas reguladoras de pesquisa envolvendo seres humanos. Resolução 196/96.

De Antoni, C. (2005). Coesão e hierarquia em famílias com histórias de abuso fisico. (Tese de Doutorado). Universidade Federal do Rio Grande do Sul, RS. (000481255)

De Antoni, C., Yunes, M. A. M., Habigzang, L., \& Koller, S. H. (2011). Abuso sexual extrafamiliar: percepções das mães de vítimas. Estudos de Psicologia, 28(1), 97-106.

Dell'Aglio, D. D., Santos, S. S., \& Borges, J. L. (2004). Infração juvenil feminina: uma trajetória de abandonos. Interação em Psicologia, 8(2), 191-198.

Dell'Aglio, D. D., Benetti, S. P., D’Incao, D. B., \& Leon, J. S. (2005). Eventos estressores no desenvolvimento de meninas adolescentes cumprindo medidas socioeducativas. Paidéia, 15(30), 119-129.

Dell'Aglio, D. D., Koller, S. H., Cerqueira-Santos, E., \& Colaço, V. F. R. (2011). Revisando o Questionário da Juventude Brasileira: uma nova proposta. In D. D. Dell'Aglio \& S. H. Koller (Orgs.), Adolescência e Juventude: vulnerabilidade e contextos de proteção (pp. 259-270). São Paulo: Casa do Psicólogo.

Espinoza-Gomez, F., Zepeda-Pamplona, V., Hernández-Bautista, V., HerándezSuárez, C. M., Newton-Sánchez, O. A., \& Plasencia-Garcia, G. R. (2010). Violencia doméstica y riesgo de conducta suicida en universitarios adolescentes. Salud Publica Mexico, 52(1), 213-219.

Fowler, P. J., Tompsett, C., Braciszewski, J., Jacques-Tiura, A., \& Baltes, B. B. (2009). Community violence: a meta-analysis on the effect of exposure and mental health outcomes of children and adolescents. Development and Psychopathology, 21, 227-259.

Guzzo, R. (2001). Saúde psicológica, sucesso escolar e eficácia na escola: desafios do novo milênio para a psicologia escolar. In Z. A. P. Del Prette (Org.), Psicologia escolar e educacional - saúde e qualidade de vida (pp.25-42). Campinas: Alínea.

Hein, A. (2004). Factores de riesgo y delincuencia juvenil: revisión de la literatura nacional e internacional. Fundación Paze Ciudadania. Recuperado de http:// www.pazciudadana.cl/docs/pub_20090623190509.pdf

Kunst, M., Winkel, F. W., \& Bogaerts, S. (2010). Prevalence and predictors of posttraumatic stress disorder among victims of violence applying for state compensation. Journal of Interpersonal Violence, 25(9), 1631-1654.

Libório, R. M. C., \& Koller, S. H. (2009). Adolescência e juventude: risco e proteção na juventude brasileira. São Paulo: Casa do Psicólogo.

Lisboa, C. S. M., \& Koller, S. H. (2004). Interações na escola e processos de aprendizagem: Fatores de risco e proteção. In A. Bzuneck, \& E. Boruchovitch (Orgs.), Aprendizagem e escola (pp. 201-224). Petrópolis: Editora Vozes.

Lisboa, C. S. M. (2005). Comportamento agressivo, vitimização e relações de amizade de crianças em idade escolar: fatores de risco e proteção. (Tese de Doutorado). Universidade Federal do Rio Grande do Sul, RS. (000536824)

Lisboa, C. S. M., Habigzang, L. F., \& Koller, S. H. (2007). Ética na pesquisa com temas delicados: estudos em psicologia com crianças e adolescentes e violência doméstica. In I. C. Z. Guerriero, M. L. S. Schmidt \& F. Zicker (Orgs.), Ética nas pesquisas em ciências humanas e sociais na saúde (pp. 176-192). São Paulo: Editora Hucitec

MacMillan, H. L., Fleming, J. E., Streiner, D. L., Lin, E., Boyle, M. H., \& Jamieson, E. (2001). Childhood abuse and lifetime psychopathology in a community sample. American Journal of Psychiatry, 158, 1878-1883.

McDonald, C. C., \& Richmond, T. R. (2008). The relationship between community violence exposure and mental health symptoms in urban adolescents. Journal of Psychiatric and Mental Health Nursing, 15, 833-849.

Meneghel, S. N., Giugliani, E. J., \& Falceto, O. (1998). Relações entre violência doméstica e agressividade na adolescência. Cadernos de Saúde Pública, 14, 327-335.

Minuchim, S., \& Fishman, H. C. (1990). Famílias. In S. Minuchim \& H. C. Fishman (Orgs.), Técnicas de terapia familiar (pp. 21-36). Porto Alegre: Artmed.

Narvaz, M., \& Oliveira, L. L. (2009). A relação entre abuso sexual e transtornos alimentares: uma revisão. Interamerican Journal of Psychology, 43(1), 22-29. 
Pesce, R. (2009). Violência familiar e comportamento agressivo e transgressor na infância: uma revisão de literatura. Ciência \& Saúde Coletiva, 14(2), 507-518.

Polanczyk, G. V., Zavaschi, M. L., Benetti, S., Zenker, R., \& Gammerman, P. W. (2003). Violência sexual e sua prevalência em adolescentes de Porto Alegre, Brasil. Revista de Saúde Pública, 37(1), 8-14.

Pratta, E. M. M., \& Santos, M. A. (2007). Família e adolescência: a influência do contexto familiar no desenvolvimento de seus membros. Psicologia em Estudo, 12(2), 247-256.

Ristum, M. (2010). Violência na escola, da escola e contra a escola. In S. G. Assis, P. Constantino \& J. Q. Avanci (Orgs.), Impactos da violência na escola: um diálogo com professores (pp. 65-93). Rio de Janeiro: Editora Fiocruz/MEC.

Sá, D. G. F., Curto, B. M., Bordin, I. A. S., \& Paula, C. S. (2009). Exposição à violência como risco para o surgimento ou a continuidade de comportamento antissocial em adolescentes da região metropolitana de São Paulo. Psicologia Teoria e Prática, 11(1), 179-188.

Salzinger, S., Feldman, R. S., Stockhammer, T., \& Hood, J. (2002). An ecological framework for understanding risk for exposure to community violence and the effects of exposure on children and adolescents. Journal of Aggression and Violent Behavior, 7, 423-451.

Santos, S. (2007). Mães de meninas que sofreram abuso sexual intrafamiliar: Reações maternas e multigeracionalidade. (Dissertação de Mestrado). Universidade Federal do Rio Grande do Sul, RS. (000602144)

Silva, E. R. A. (2004). O direito à convivência familiar e comunitária: os abrigos para crianças e adolescentes no Brasil: Brasília: IPEA/CONANDA.

Siqueira, A. C., Betts, M. K., \& Dell'Aglio, D. D. (2006). A rede de apoio social e afetivo de adolescentes institucionalizados no sul do Brasil. Revista Interamericana de Psicologia, 40(2), 149-158.

Spano, R., Rivera, C., \& Bolland, J. M. (2010). Does parenting shield youth from exposure to violence during adolescence? A 5-year longitudinal test in a highpoverty sample of minority youth. Journal of Interpersonal Violence, 7, 1-20.

Steinberg, L. (2000). The family at adolescence: transition and transformation. Journal of Adolescent Health, 27, 170-178.

Sullca, T. F., \& Schirmer, J. (2006). Violência intrafamiliar na adolescência na cidade de Puno - Peru. Revista Latino Americana de Enfermagem, 14(4), 578-585.

Sullivan, T., Farrell, S., Kliewer, W., Vulin-Reynolds, M., \& Valois, R. (2007). Exposure to violence in early adolescence: the impact of self-restraint, witnessing violence, and victimization on aggression and drug use. Journal of Early Adolescence, 27(3), 296-323.

Vasconcelos, M. M., Malheiros, A. F. A., Werner Jr., J., Brito, A. R., Barbosa, J. B., Santos, I. S. O., \& Lima, D. F. N. (2005). Contribuição dos fatores de risco psicossociais para o Transtorno de Déficit de Atenção/hiperatividade. Arquivos de Neuro-Psiquiatria, 63(1), 68-74.

World Health Organization (2010). Participant manual - IMAI One-day Orientation on Adolescents Living with HIV Geneva. Recuperado de http:// whqlibdoc.who.int/publications/2010/9789241598972_eng.pdf

Luiza Lima Braga, mestre em Psicologia pela Universidade Federal do Rio Grande do Sul (UFRGS). Endereço para correspondência: Universidade Federal do Rio Grande do Sul, Instituto de Psicologia, Departamento de Psicologia do Desenvolvimento e da Personalidade.

Rua Ramiro Barcelos, 2600/ sala 115. CEP: 90035-003 - Porto Alegre, RS. E-mail: luiza.lima.braga@ gmail.com

Débora Dalbosco Dell'Aglio, doutora em Psicologia do Desenvolvimento pela Universidade Federal do Rio Grande do Sul (UFRGS), é docente do Programa de Pós-Graduação em Psicologia da Universidade Federal do Rio Grande do Sul (UFRGS). E-mail: dalbosco@cpovo.net 